\title{
Prevalence of Carbapenem-Resistant Enterobacteriaceae in Seoul, Korea
}

\section{Sang-Hun Park*, Jin-Seok Kim, Hee-Soon Kim, Jin-Kyung Yu, Sung-Hee Han, Min-Ji Kang, Chae-Kyu Hong, Sang-Me Lee, Young-Hee Oh}

Seoul Metropolitan Government Research Institute of Public Health and Environment, 30, Janggunmaeul 3-gil, Gwacheon-si, Gyeonggi-do 13818, Republic of Korea

\author{
Corresponding \\ Sang-Hun Park, Researcher \\ Seoul Metropolitan Government \\ Research Institute of Public Health and \\ Environment, 30, Janggunmaeul 3-gil, \\ Gwacheon-si, Gyeonggi-do 13818, \\ Republic of Korea \\ Phone : +82-02-570-3419 \\ Fax : $+82-02-570-3418$ \\ E-mail : sanghun93@seoul.go.kr
}

Received : April 22, 2020

Revised : May 26, 2020

Accepted : May 29, 2020

No potential conflict of interest relevant to this article was reported.

Copyright (C) 2020 Journal of Bacteriology and Virology

(C) This is an Open Access article distributed under the terms of the Creative Commons Attribution Non-Commercial License

(http://creativecommons.org/ license/by-nc/3.0/).
The prevalence of carbapenem-resistant Enterobacteriaceae (CRE) is increasing globally. However, a few studies have addressed their epidemiology in Seoul, Korea. In this study, we conducted one-year surveillance of CRE among 1,468 clinical isolates of Enterobacteriaceae at the hospital in Seoul with molecular characterization of carbapenemase genes. About $85 \%$ of CRE-positive samples were isolated from the elderly age group (above 60 years). The most common isolated organisms were Klebsiella pneumoniae (K. pneumoniae) (56.5\%) and Escherichia coli (E.coli) $(17.0 \%)$. We detected six different Carbapenemase-producing Enterobacteriaceae

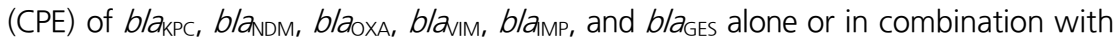
other bla genes. Typically, $853(58.1 \%)$ isolates were tested positive for at least one CPE. KPC ( $K$. pneumoniae carbapenemase)-2 was the most common CPE type (46.0\%) followed by NDM (New Delhi metallo- $\beta$-lactamase)-1 (5.9\%). KPC-2 was most commonly found in K. pneumoniae (494/676 isolates [73.1\%]) and E.coli (107/676 isolates [15.8\%]), whereas NDM-1 was commonly found in Enterobacter cloacae complex (20/86 isolates [23.3\%]). Detailed information and molecular characteristics of CPE is essential to prevent the spread of these pathogens.

Key Words: Carbapenem-resistant Enterobacteriaceae (CRE), Carbapenemaseproducing Enterobacteriaceae (CPE), Klebsiella pneumoniae

\section{INTRODUCTION}

Carbapenem-resistant Enterobacteriaceae (CRE) is an emerging problematic infectious agent, with reports of its prevalence worldwide (1-3). CRE outbreak in hospitals has become a critical issue. Transmission by patients with CRE, as well as carriers of CRE, contribute significantly to in-hospital CRE transmission (4). Carbapenemase-producing Enterobacteriaceae (CPE) are an important and increasing threat to global health (5). Infections due to these organisms are associated with significant morbidity and mortality (1).

CPE produce enzymes that fall into three classes according to the Ambler classification: class A $\beta$-lactamases (Klebsiella pneumoniae carbapenemase [KPC]), class B metallo- $\beta$-lactamases (New Delhi metallo- $\beta$-lactamase [NDM], imipenemase [IMP], and Verona integron-encoded metallo- $\beta$-lactamase [VIM]), and class D $\beta$-lactamases (oxacillinase [OXA]-48). The carbapenemase genes in Enterobacteriaceae have been shown to be associated with mobile genetic elements such as plasmids or transposons, thereby facilitating infection outbreaks (6). 
An understanding of the epidemiology of the emergence of CPE and the changing burden over time is critical to the implementation of control programs and the management of individual patients (7). The purpose of this study is to investigate the prevalence and molecular epidemiology of CPE isolates collected from Seoul area in 2018.

\section{MATERIALS AND METHODS}

\section{Bacterial strains}

From January 2018 to December 2018, a total of 1,468 CRE clinical isolates were retrospectively collected from 113 hospitals in Seoul (Korea). Isolation sites were blood, urine, stool, sputum, lesion, bile, pus, tracheal aspiration, and etc. Bacterial identification was performed using Bruker Biotyper MALDI-TOF MS (Bruker Daltonics, Bremen, Germany) and VITEK 2 (bioMérieux, Marcy l'Etoile, France).

\section{Antimicrobial susceptibility testing}

Antimicrobial susceptibility testing was carried out by broth microdilution using customized Sensititre plates (TREK Diagnostic Systems, Cleveland, OH, USA). CRE were found to be resistant to imipenem, meropenem, doripenem, and ertapenem based on the Clinical and Laboratory Standards Institute (CLSI) guidelines (8).

\section{PCR detection and sequencing of CPE genes}

PCR and subsequent sequencing were conducted to detect and identify the main CPE (blakPC and blaGES from class A; blamp, blavim, and blandM from class B; and blaoxA from class D)-encoding genes, as previously described (9) (Table 1). Briefly, the template DNAs were prepared by boiling, and PCR amplification was conducted using Quick Taq HS DyeMix (TOYOBO, Japan) with specific primer pairs. The PCR conditions were: $94^{\circ} \mathrm{C}$ for $5 \mathrm{~min}$, followed by 30 cycles of

Table 1. Primer pairs used for amplification of CPE

\begin{tabular}{|c|c|c|}
\hline Target genes & Sequences $\left(5^{\prime} \rightarrow 3^{\prime}\right)$ & Product size $(b p)$ \\
\hline \multirow{2}{*}{ IMP } & F TGA GCA ATG TAT CTG TAT TC & \multirow{2}{*}{740} \\
\hline & R TTA GTT GCT TGG TTT TGA TG & \\
\hline \multirow{2}{*}{ OXA-48 } & F TTG GTG GCA TCG ATT ATC GG & \multirow{2}{*}{743} \\
\hline & R GAG CAC TTC TTT TGT GAT GGC & \\
\hline \multirow{2}{*}{ VIM } & F TGG TCT ACA TGA CCG CGT CT & \multirow{2}{*}{766} \\
\hline & R CGA CTG AGC GAT TTG TGT G & \\
\hline \multirow{2}{*}{ NDM } & F CAA TAT TAT GCA CCC GGT CG & \multirow{2}{*}{720} \\
\hline & R ATC ATG CTG GCC TTG GGG AA & \\
\hline \multirow{2}{*}{$\mathrm{KPC}$} & F ATG TCA CTG TAT CGC CGT CT & \multirow{2}{*}{893} \\
\hline & R TTT TCA GAG CCT TAC TGC CC & \\
\hline \multirow{2}{*}{ GES } & F GCG CTT CAT TCA CGC ACT AT & \multirow{2}{*}{753} \\
\hline & R GCG TAA TCT CTC TCC TGG GC & \\
\hline \multirow{2}{*}{ SME } & F AAC GGC TTC ATT TTT G & \multirow{2}{*}{830} \\
\hline & R GCT TCC GCA ATA GTT TTA TCA & \\
\hline \multirow{2}{*}{ GIM } & F TCG ACA CAC CTT GGT CTG AA & \multirow{2}{*}{477} \\
\hline & R AAC TTC CAA CTT TGC CAT GC & \\
\hline \multirow{2}{*}{ SIM } & F TAC AAG GGA TTC GGC ATC G & \multirow{2}{*}{570} \\
\hline & R TAA TGG CCT GTT CCC ATG TG & \\
\hline \multirow{2}{*}{ SPM } & F AAA ATC TGG GTA CTC AAA CG & \multirow{2}{*}{271} \\
\hline & R ACA TTA TCC GCT GGA ACA GG & \\
\hline
\end{tabular}


denaturation at $94^{\circ} \mathrm{C}$ for $30 \mathrm{~s}$, annealing at $56^{\circ} \mathrm{C}$ for $20 \mathrm{~s}$ and elongation at $72^{\circ} \mathrm{C}$ for $30 \mathrm{~s}$; and a final extension at $72^{\circ} \mathrm{C}$ for 7 min. PCR products were purified using a QIAquick Gel Extraction Kit (Qiagen, Hilden, Germany). Analyses of nucleotide sequences were carried out by BIOFACT Co. (Daejeon, Korea). Sequences were compared with available sequences in GenBank using the BLAST program of the National Center for Biotechnology Information (NCBI).

\section{RESULTS}

\section{CRE isolates and their resistance characteristics}

While stratifying all CRE isolates by age group, it was observed that 407 (27.7\%) isolates were from $70-79$ years age group, 392 (26.7\%) were from 80-89 years age group, 252 (17.2\%) were from 60-69 years age group, 138 (9.4\%) were from 50-59 years age group, 58 (4.0\%) were from 40-49 years age group, 55 (3.7\%) were from above 90 years age group, 49 (3.3\%) were from 0-9 years age group, 41 (2.8\%) were from 30-39 years age group, 20 (1.4\%) were from 20-29 years age group, and $6(0.4 \%)$ were from 10-19 years age group (Table 2). For all the analyzed isolates from patients, 806 (54.9\%) were from male patients and 589 (40.1\%) were from female patients (Table 2). The types of CRE isolates are shown in Table 3. Of 1,468 study isolates, Klebsiella pneumoniae (K. pneumoniae) was the most common isolated CRE organism in this study (830 isolates, 56.5\%) followed by Escherichia coli (E. col/) (249 isolates, 17\%), Enterobacter cloacae complex (136 isolates, 9.3\%), Klebsiella aerogenes (63 isolates, 4.3\%), Proteus mirabilis (41 isolates, $2.8 \%)$, Citrobacter koseri (37 isolates, 2.5\%), and Citrobacter freundii (34 isolates, $2.3 \%$ ).

\section{Genetic characterization of carbapenemases genes among CRE isolates}

The distribution of different carbapenemases among the Enterobacteriaceae isolates is summarized in Table 4. Of 1,468 samples tested, $853(58.1 \%)$ were tested positive for at least one CPE. KPC-2 was the most common CPE type (46.0\%), followed by NDM-1 (5.9\%), NDM-5 (1.8\%), NDM-5 co-infected with OXA-181 (0.8\%), OXA-181 (0.7\%), OXA-232 (0.5\%), NDM-4 $(0.5 \%)$, OXA-48 $(0.3 \%), \mathrm{VIM}-2(0.3 \%)$, and NDM-1 with OXA-232 $(0.2 \%)$. Also, a relatively low detection frequency of IMP-1, KPC-2 with NDM-1, KPC-2 with GES-5, KPC-4, and NDM-13, KPC-4 with NDM-1, OXA-48 with NDM-5, IMP-6, GES-5, and GES-5 with VIM-2 at $0.1 \%$ was observed, respectively. KPC-2 was most commonly found in K. pneumoniae (494/676 isolates [73.1\%]) and E. coli (107/676 isolates [15.8\%]), whereas NDM-1 was mostly found in Enterobacter cloacae complex (20/86 isolates [23.3\%]) (Table 5).

Table 2. Distribution of CRE by gender and age group

\begin{tabular}{ccccc}
\hline Age & No. of male $(\%)$ & No. of female $(\%)$ & No. of unknown $(\%)$ & Total $(\%)$ \\
\hline $0-9$ & $24(1.6)$ & $23(1.6)$ & $2(0.1)$ & $49(3.3)$ \\
$10-19$ & $5(0.3)$ & $1(0.1)$ & $0(0.0)$ & $6(0.4)$ \\
$20-29$ & $12(0.8)$ & $6(0.4)$ & $2(0.1)$ & $20(1.4)$ \\
$30-39$ & $17(1.2)$ & $23(1.6)$ & $1(0.1)$ & $41(2.8)$ \\
$40-49$ & $41(2.8)$ & $17(1.2)$ & $0(0.0)$ & $58(4.0)$ \\
$50-59$ & $95(6.5)$ & $39(2.7)$ & $4(0.3)$ & $138(9.4)$ \\
$60-69$ & $161(11.0)$ & $82(5.6)$ & $9(0.6)$ & $252(17.2)$ \\
$70-79$ & $247(16.8)$ & $149(10.1)$ & $11(0.7)$ & $407(27.7)$ \\
$80-89$ & $177(12.1)$ & $208(14.2)$ & $7(0.5)$ & $392(26.7)$ \\
$>=90$ & $21(1.4)$ & $34(2.3)$ & $0(0.0)$ & $55(3.7)$ \\
Unknown & $6(0.4)$ & $7(0.5)$ & $37(2.5)$ & $50(3.4)$ \\
Total & $806(54.9)$ & $589(40.1)$ & $73(5.0)$ & $1.468(100.0)$ \\
\hline
\end{tabular}


Table 3. Distribution of species in CRE isolates

\begin{tabular}{ccc}
\hline Types of isolates & No. of isolates & Percentage (\%) \\
\hline Klebsiella pneumoniae & 830 & 56.5 \\
Escherichia coli & 249 & 17.0 \\
Enterobacter cloacae complex & 136 & 9.3 \\
Klebsiella aerogenes & 63 & 4.3 \\
Proteus mirabilis & 41 & 2.8 \\
Citrobacter koseri & 37 & 2.5 \\
Citrobacter freundii & 34 & 2.3 \\
Serratia marcescens & 15 & 1.0 \\
Klebsiella oxytoca & 15 & 1.0 \\
Providencia rettgeri & 14 & 1.0 \\
Morganella morganii & 8 & 0.5 \\
Proteus vulgaris & 8 & 0.5 \\
Citrobacter braakii & 4 & 0.3 \\
Raoultella ornithinolytica & 3 & 0.2 \\
Providencia stuartii & 2 & 0.1 \\
Citrobacter amalonaticus & 1 & 0.1 \\
Hafnia alvei & 1 & 0.1 \\
Citrobacter youngae & 1 & 0.1 \\
Escherichia hermannii & 1 & 0.1 \\
Kluyvera cryocrescens & 1 & 0.1 \\
Citrobacter farmeri & 1 & 0.1 \\
Kluyvera intermedia & 1 & 0.1 \\
Raoultella planticola & 1 & 0.1 \\
Proteus penneri & 1 & 0.1 \\
Total & 1,468 & 100 \\
\hline
\end{tabular}

Table 4. Distribution of carbapenemase genotypes by CRE isolates $(n=1,468)$

\begin{tabular}{ccc}
\hline Types of isolates & No. of isolates & Percentage (\%) \\
\hline KPC-2 & 676 & 46.0 \\
NDM-1 & 86 & 5.9 \\
NDM-5 & 26 & 1.8 \\
NDM-5, OXA-181 & 12 & 0.8 \\
OXA-181 & 11 & 0.7 \\
OXA-232 & 8 & 0.5 \\
NDM-4 & 8 & 0.5 \\
OXA-48 & 5 & 0.3 \\
VIM-2 & 4 & 0.3 \\
NDM-1, OXA-232 & 3 & 0.2 \\
IMP-4 & 2 & 0.1 \\
NDM-4, OXA-181 & 2 & 0.1 \\
IMP-1 & 1 & 0.1 \\
KPC-2, NDM-1 & 1 & 0.1 \\
KPC-2, GES-5 & 1 & 0.1 \\
KPC-4 & 1 & 0.1 \\
NDM-13 & 1 & 0.1 \\
KPC-4, NDM-1 & 1 & 0.1 \\
OXA-48, NDM-5 & 1 & 0.1 \\
IMP-6 & 1 & 0.1 \\
GES-5 & 1 & 0.1 \\
GES-5, VIM-2 & 1 & 0.1 \\
NEG* & 615 & 41.9 \\
Total & 1,468 & 100 \\
\hline
\end{tabular}

*carbapenemase gene negative 


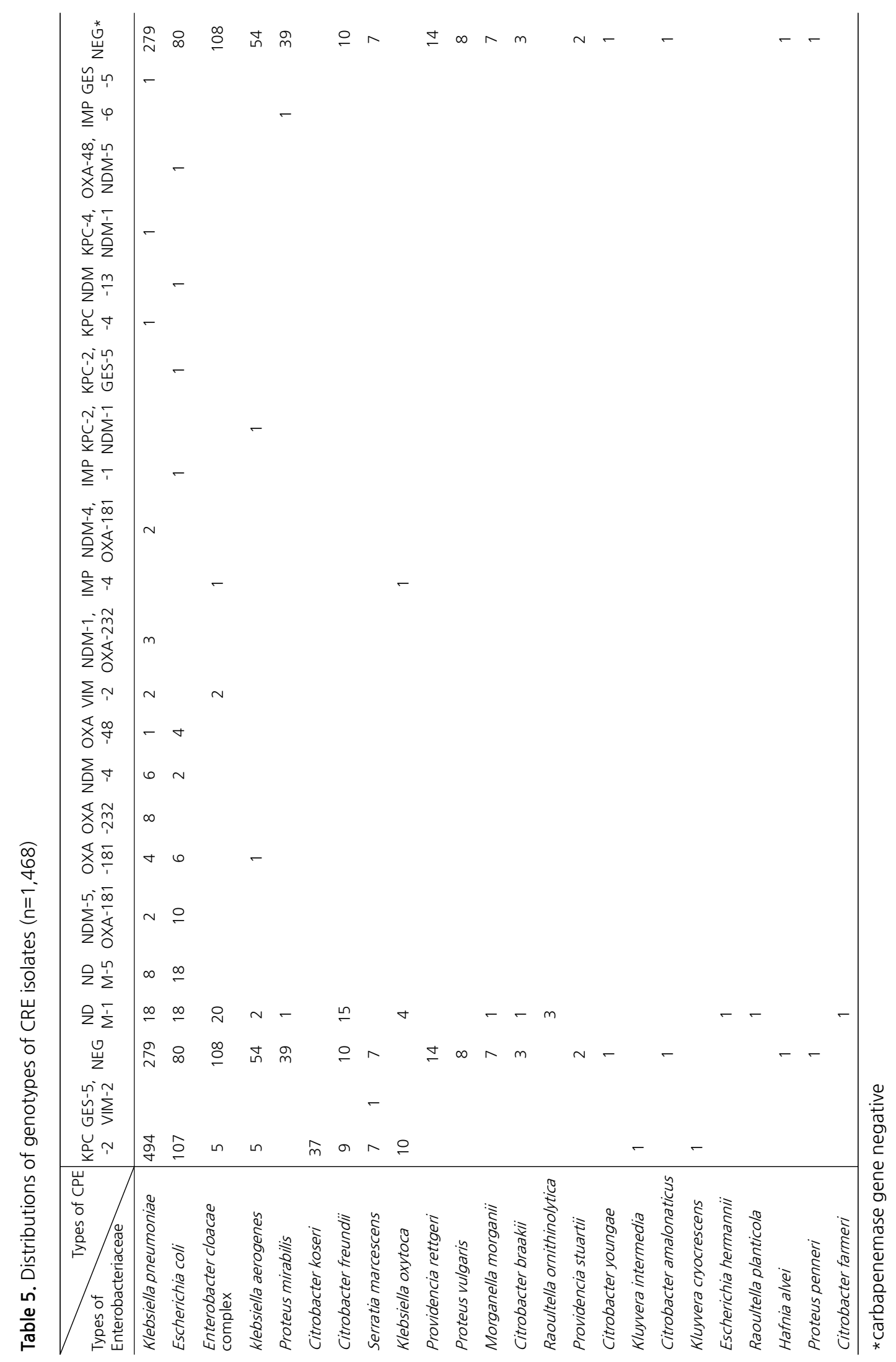




\section{DISCUSSION}

CRE is of significant concern to public health, both at the community level and for the health-care facilities (10). The worldwide global increase in infections with CRE is of great concern due to the association of infections with the highly virulent bacteria with high morbidity and mortality rates (10). Until now, the carbapenem resistance rate among Enterobacteriaceae isolates from Korea has been relatively low and stable (11).

When we considered CRE isolates, the age distribution was skewed toward older patients, accounting for $85 \%$ of isolates (above 60 years of age).

Our findings that K. pneumoniae was the dominant type (identified in $56.5 \%$ of isolated), followed by $E$. coli (only $17.0 \%$ ) is in agreement with those of previous studies done in community hospitals in Bahrain, Taiwan, and US where $K$. pneumoniae was the most prevalent species $(91 \%)(10,12,13)$. K. pneumoniae will play a major role in carbapenem resistance because it has been repeatedly identified to be the most common species among CRE, hereafter called carbapenem-resistant K. pneumoniae (CRKP) (14).

It was found that the emergence of CRE has become a formidable public health threat as it had increased four-fold over the past 10 years worldwide (15), particularly among K. pneumoniae and E. coli, as has been reported in the global antibiotic resistance estimates published by the World Health Organization in 2014 (16). The results of the present study on the leading causes of CRE were similar to those of previous studies that focused on community hospitals. However, the prevalence rate of $E$. coli and $K$. pneumoniae was either on a little lower or higher side than the rate specified in previous reports. This may be due to the difference between countries and the reporting years $(10,17,18)$.

Three main carbapenemases are reported worldwide: KPC, NDM, and OXA-48-like (18). KPC the most common transmissible class A genes are mostly found worldwide $(19,20)$. KPC-2, NDM-1, NDM-5, and OXA-181 are the most common carbapenemases identified in the present study, and the outcome is similar to previous findings describing the detection of KPC, NDM, and OXA-48 worldwide $(21,22)$. Korea is known for outbreaks of $K$. pneumoniae that produce KPC-2, NDM-1, and OXA-232 $(20,23,24)$. After the first KPC producer (KPC-2 in K. pneumoniae) was identified in 1996 in the eastern United States (25), reports of KPC-2 in the New York, NY, area began to appear in 2004; KPC-expressing $K$. pneumoniae is currently an alarming problem (26-28). This report is especially disturbing because New York demonstrated large outbreaks of extended-spectrum $\beta$-lactamase (ESBL)-producing Klebsiella $(29,30)$ for which carbapenems were considered to be one of the few treatment options (16). Since numerous studies on KPC-2 genes have been carried out worldwide $(31,32)$, we need to carefully monitor CPE and perform in-depth research on CPE using surveillance systems.

Previous studies indicated increasing numbers of Enterobacteriaceae as frequent carriers of genes encoding two of the most concerning subclasses of carbapenemases: KPC, which has become endemic in parts of America, Southern Europe, Israel, and China; and the New Delhi Metallo- $\beta$-lactamase (NDM), which has become endemic in Northern Europe and the Asia Pacific region, most remarkably in the UK and India (33-35). Mechanisms of carbapenem nonsusceptibility can be divided broadly into carbapenemase production CPE and a combination of $\beta$-lactamase (ESBL and AmpC) production with porin loss, and efflux pump overexpression (non-carbapenemase-producing carbapenem nonsusceptible Enterobacteriaceae [NCPCRE]) (36). These mechanisms generally appear paired among themselves or with carbapenemase-production (1). All three alternative mechanisms aim to block the penetration of the antibiotics within the bacterial cell (37). The data from a case-control study comparing patients with NCPCRE (cases) and patients with CPE (controls) reported in the United States indicate 843 unique patients with first-episode CRE, including 387 (45.9\%) NCPCRE and 456 (54.1\%) CPE (38). Orsi and colleagues (39) demonstrated that compared to Klebsiella pneumoniae carbapenemase (KPC)-producing CRE, NCPCRE were associated with prior antibiotic exposure, demonstrating that patient-level risk factors may differ according to mechanisms of resistance. Another study performed in Taiwan from 2010 to 2012 presented the detection of Carbapenemase in $5.0 \%$ of the CRE isolates but the prevalence and carbapenemase differed by species (40). 
Although KPC is the most common carbapenemase worldwide, NDM has also been shown to be important sporadic outbreaks around the globe $(41,42)$. The prevalence of NDM was in agreement with a study on Global distribution (43). The analysis of a total of 1,468 isolates of Enterobacteriaceae for the presence of CPE demonstrated NDM as the second gene $(9.6 \%, 141 / 1,468)$ and mostly NDM-1 $(64.5 \%, 91 / 141)$ in Enterobacteriaceae. CPE distribution likely appears to vary geographically; for example, high rates in Greece (VIM and KPC), Romania, Poland and Denmark (NDM), and in the Indian subcontinent (NDM, KPC, and OXA-181) (44, 45). MBL-producing Enterobacteriaceae isolates have also been reported in several Latin American countries and Europe (45). In a multinational survey spanning 2012-2014, VIM-producing CPE were recovered in Mexico and NDM-1-producing CPE in Venezuela (45).

CRE continues to evolve, posing an increasing threat to patients of all ages (1). Early identification of carbapenemase producers in clinical infections, at the carriage state, or both, is therefore mandatory to prevent the development of hospital-based outbreaks (44). Consequently, it is hypothesized that constant surveillance and regular education are important for the successful mitigation of health-care-associated infection and to decrease the incidence rate of CRE. Molecular characterization of CPE is essential for epidemiological surveillance to monitor the insurgence of the CPE pandemic.

In conclusion, this study revealed the overall prevalence of CRE in Seoul, Korea and highlights the need for continued studies as a way to control the emergence of new CPE varieties as a basis for further epidemiological surveillance

\section{CONFLICT OF INTEREST}

The authors declare that the research was conducted in the absence of any commercial or financial relationships that could be constructed as a potential conflict of interest.

\section{REFERENCES}

1) Logan LK, Weinstein RA. The Epidemiology of Carbapenem-Resistant Enterobacteriaceae: The Impact and Evolution of a Global Menace. J Infect Dis 2017;215:S28-S36.

2) Livorsi DJ, Chorazy ML, Schweizer ML, Balkenende EC, Blevins $A E$, Nair R, et al. A systematic review of the epidemiology of carbapenem-resistant Enterobacteriaceae in the United States. Antimicrob Resist Infect Control 2018;7:55.

3) Zhang Y, Wang Q, Yin Y, Chen H, Jin L, Gu B, et al. Epidemiology of carbapenem-resistant Enterobacteriaceae infections: report from the China CRE network. Antimicrob Agents Chemother 2018;62:e01882-17.

4) Lerner A, Adler A, Abu-Hanna J, Meitus I, Navon-Venezia S, Carmeli Y. Environmental contamination by carbapenem-resistant Enterobacteriaceae. J Clin Microbio/2013;51:177-81.

5) van Duin D, Doi Y. The global epidemiology of carbapenemase-producing Enterobacteriaceae. Virulence 2017:8:460-9.

6) Queenan AM, Bush K. Carbapenemases: the versatile beta-lactamases. Clin Microbio/ Rev 2007:20:440-58.

7) Kohler PP, Melano RG, Patel SN, Shafinaz S, Faheem A, Coleman BL, et al. Emergence of Carbapenemase-Producing Enterobacteriaceae, South-Central Ontario, Canada. Emerg Infect Dis 2018:24:1674-82.

8) Clinical and Laboratory Standards Institute (CLSI). Performance Standards for Antimicrobial Susceptibility Testing M100-S26. Wayne, PA: CLSI, 2016. 
9) Kim JS, Jin YH, Park SH, Han S, Kim HS, Park JH, et al. Emergence of a multidrug-resistant clinical isolate of Escherichia coliST8499 strain producing NDM-13 carbapenemase in the Republic of Korea. Diagn Microbiol Infect Dis 2019:94:410-2.

10) Saeed NK, Alkhawaja S, Azam NFAEM, Alaradi K, Al-Biltagi M. Epidemiology of carbapenem-resistant Enterobacteriaceae in a Tertiary Care Center in the Kingdom of Bahrain. J Lab Physicians 2019;11:111-7.

11) Korea Centers for Disease Control and Prevention (KCDC). Korean Antimicrobial Resistance Monitoring System (KARMS) annual report, 2014. Korea Centers for Disease Control and Prevention, 2015.

12) Jean SS, Lee NY, Tang HJ, Lu MC, Ko WC, Hsueh PR. Carbapenem-Resistant Enterobacteriaceae Infections: Taiwan Aspects. Front Microbio/2018;9:2888.

13) Thaden JT, Lewis SS, Hazen KC, Huslage K, Fowler VG Jr, Moehring RW, et al. Rising rates of carbapenem-resistant Enterobacteriaceae in community hospitals: A mixed-methods review of epidemiology and microbiology practices in a network of community hospitals in the Southeastern United States. Infect Control Hosp Epidemio/2014;35:978-83.

14) Netikul T, Kiratisin P. Genetic Characterization of Carbapenem-Resistant Enterobacteriaceae and the Spread of Carbapenem-Resistant Klebsiella pneumonia ST340 at a University Hospital in Thailand. PLoS One 2015; 10:e0139116.

15) Weiner LM, Webb AK, Limbago B, Dudeck MA, Patel J, Kallen AJ, et al. Antimicrobial-resistant pathogens associated with healthcare-associated infections: Summary of data reported to the national healthcare safety network at the centers for disease control and prevention, 2011-2014. Infect Control Hosp Epidemio/2016;37:1288-301.

16) Paterson DL, Bonomo RA. Extended-spectrum beta-lactamases: a clinical update. Clin Microbio/ Rev 2005;18:657-86.

17) Pan F, Tian D, Wang B, Zhao W, Qin H, Zhang T, et al. Fecal carriage and molecular epidemiology of carbapenem-resistant Enterobacteriaceae from outpatient children in Shanghai. BMC Infect Dis 2019; $19: 678$.

18) Rizzo K, Horwich-Scholefield S, Epson E. Carbapenem and Cephalosporin Resistance among Enterobacteriaceae in Healthcare-Associated Infections, California, USA. Emerg Infect Dis 2019;25:1389-93.

19) Patel G, Bonomo RA. "Stormy waters ahead": global emergence of carbapenemases. Front Microbio/2013;4:48.

20) Jeong SH, Kim HS, Kim JS, Shin DH, Kim HS, Park MJ, et al. Prevalence and Molecular Characteristics of Carbapenemase-Producing Enterobacteriaceae From Five Hospitals in Korea. Ann Lab Med 2016;36:529-35.

21) Nordmann P, Poirel L. The difficult-to-control spread of carbapenemase producers among Enterobacteriaceae worldwide. Clin Microbiol Infect 2014;20:821-30.

22) Sugawara E, Kojima S, Nikaido H. Klebsiella pneumoniae Major Porins OmpK35 and OmpK36 allow more efficient diffusion of beta-lactams than their Escherichia colihomologs OmpF and OmpC. J Bacterio/2016;198:3200-8.

23) Kim MN, Yong D, An D, Chung HS, Woo JH, Lee K, et al. Nosocomial clustering of NDM-1-producing Klebsiella pneumoniae sequence type 340 strains in four patients at a South Korean tertiary care hospital. J Clin Microbiol 2012:50:1433-6.

24) Hong SK, Yong D, Kim K, Hong SS, Hong SG, Khosbayar T, et al. First outbreak of KPC-2-producing Klebsiella pneumoniae sequence type 258 in a hospital in South Korea. J Clin Microbio/2013;51:3877-9.

25) Yigit H, Queenan AM, Anderson GJ, Domenech-Sanchez A, Biddle JW, Steward CD, et al. Novel carbapenemhydrolyzing beta-lactamase, KPC-1, from a carbapenem-resistant strain of Klebsiella pneumoniae. Antimicrob Agents Chemother 2001;45:1151-61. 
26) Bradford PA, Bratu S, Urban C, Visalli M, Mariano N, Landman D, et al. Emergence of carbapenem-resistant Klebsiella species possessing the class A carbapenem-hydrolyzing KPC-2 and inhibitor-resistant TEM-30 beta-lactamases in New York City. Clin Infect Dis 2004;39:55-60.

27) Bratu S, Landman D, Haag R, Recco R, Eramo A, Alam M, et al. Rapid spread of carbapenem-resistant Klebsiella pneumoniae in New York City: a new threat to our antibiotic armamentarium. Arch Intern Med 2005;165:1430-5.

28) Bratu S, Tolaney P, Karumudi U, Quale J, Mooty M, Nichani S, et al. Carbapenemase-producing Klebsiella pneumoniae in Brooklyn, NY: molecular epidemiology and in vitro activity of polymyxin B and other agents. $J$ Antimicrob Chemother 2005:56:128-32.

29) Meyer KS, Urban C, Eagan JA, Berger BJ, Rahal JJ. Nosocomial outbreak of Klebsiella infection resistant to late-generation cephalosporins. Ann Intern Med 1993;119:353-8.

30) Quale JM, Landman D, Bradford PA, Visalli M, Ravishankar J, Flores C, et al. Molecular epidemiology of a citywide outbreak of extended-spectrum beta-lactamase-producing Klebsiella pneumoniae infection. Clin Infect Dis 2002:35:834-41.

31) Nordmann P, Cuzon G, Naas T. The real threat of Klebsiella pneumoniae carbapenemase-producing bacteria. Lancet Infect Dis 2009:9:228-36.

32) Navon-Venezia S, Chmelnitsky I, Leavitt A, Schwaber MJ, Schwartz D, Carmeli Y. Plasmid-mediated imipenemhydrolyzing enzyme KPC-2 among multiple carbapenem-resistant Escherichia coli clones in Israel. Antimicrob Agents Chemother 2006:50:3098-101.

33) National Institute of Allergy and Infectious Diseases (NIAID). NIAID's Antibacterial Resistance Program: current status and future directions 2014. https://www.niaid.nih.gov/sites/default/files/arstrategicplan2014.pdf

34) Bush K. Proliferation and significance of clinically relevant $\beta$-lactamases. Ann N Y Acad Sci 2013;1277:84-90.

35) Schwaber MJ, Lev B, Israeli A, Solter E, Smollan G, Rubinovitch B, et al. Containment of a country-wide outbreak of carbapenem-resistant Klebsiella pneumoniae in Israeli hospitals via a nationally implemented intervention. Clin Infect Dis 2011;52:848-55.

36) Goodman KE, Simner PJ, Tamma PD, Milstone AM. Infection control implications of heterogeneous resistance mechanisms in carbapenem-resistant Enterobacteriaceae (CRE). Expert Rev Anti Infect Ther 2016;14:95-108.

37) Suay-García B, Pérez-Gracia MT. Present and Future of Carbapenem-resistant Enterobacteriaceae (CRE) Infections. Antibiotics (Basel). 2019;8:122.

38) Marimuthu K, Ng OT, Cherng BPZ, Fong RKC, Pada SK, De PP, et al. Antecedent Carbapenem Exposure as a Risk Factor for Non-Carbapenemase-Producing Carbapenem-Resistant Enterobacteriaceae and Carbapenemase-Producing Enterobacteriaceae. Antimicrob Agents Chemother 2019;63:e00845-19.

39) Orsi GB, Bencardino A, Vena A, Carattoli A, Venditti C, Falcone M, et al. Patient risk factors for outer membrane permeability and KPC-producing carbapenem-resistant Klebsiella pneumoniae isolation: results of a double casecontrol study. Infection 2013;41:61-7.

40) Wang JT, Wu UI, Lauderdale TL, Chen MC, Li SY, Hsu LY, et al. Carbapenem-nonsusceptible Enterobacteriaceae in Taiwan. PLoS One 2015;10:e0121668.

41) Kim Y, Cunningham MA, Mire J, Tesar C, Sacchettini J, Joachimiak A. NDM-1, the ultimate promiscuous enzyme: substrate recognition and catalytic mechanism. FASEB J 2013;27:1917-27. 
42) Zmarlicka MT, Nailor MD, Nicolau DP. Impact of the New Delhi metallo-beta-lactamase on beta-lactam antibiotics. Infect Drug Resist 2015; 8:297-309.

43) Kazmierczak KM, Rabine S, Hackel M, McLaughlin RE, Biedenbach DJ, Bouchillon SK, et al. Multiyear, multinational survey of the incidence and global distribution of metallo-beta-lactamase-producing Enterobacteriaceae and Pseudomonas aeruginosa. Antimicrob Agents Chemother 2015:60:1067-78.

44) Nordmann P, Naas T, Poirel L. Global spread of Carbapenemase-producing Enterobacteriaceae. Emerg Infect Dis 2011:17:1791-8.

45) Albiger B, Glasner C, Struelens MJ, Grundmann H, Monnet DL. Carbapenemase-producing Enterobacteriaceae in Europe: assessment by national experts from 38 countries, May 2015. Euro Surveill 2015;20. 